\title{
GEOMETRIC CONVERGENCE OF THE KÄHLER-RICCI FLOW ON COMPLEX SURFACES OF GENERAL TYPE
}

\author{
BIN GUO, JIAN SONG, AND BEN WEINKOVE
}

\begin{abstract}
We show that on smooth minimal surfaces of general type, the Kähler-Ricci flow starting at any initial Kähler metric converges in the Gromov-Hausdorff sense to a Kähler-Einstein orbifold surface. In particular, the diameter of the evolving metrics is uniformly bounded for all time and the Kähler-Ricci flow contracts all the holomorphic spheres with $(-2)$ self-intersection number to isolated orbifold points. Our estimates do not require a priori the existence of an orbifold KählerEinstein metric on the canonical model.
\end{abstract}

\section{INTRODUCTION}

The Ricci flow, introduced by Hamilton [H] has become a powerful tool to study the topology and geometric structures of Riemannian manifolds. In general, the Ricci flow develops finite time singularities. Hamilton's program of Ricci flow with surgeries was carried out by Perelman [P1, [P2, [P3] to prove Thurston's geometrization conjecture. The minimal model theory in birational geometry can be viewed as the complex analogue of Thurston's geometrization conjecture. It was proposed in [SoT3 that the Ricci flow will carry out an analytic minimal model program on projective varieties, analogous to the 3-dimensional Ricci flow with surgeries.

In particular, it was conjectured in [SoT3] that the Ricci flow will perform a canonical surgery, corresponding to a birational transformation such as a divisorial contraction or flip, whenever it encounters a non-volume-collapsing singularity at finite time (see also [T] ). This extended earlier conjectures of [FIK], which were established in [SW1, CT]. The theory of the weak KählerRicci flow was developed in [SoT3], where it was shown that the flow can be continued through these birational transformations at the level of potentials. These surgeries are conjectured [SoT3] to be continuous in the GromovHausdorff topology, and this was confirmed for Kähler surfaces [SW2, SW3, SW4. Indeed it was shown that if the total volume is uniformly bounded below away from zero at a finite time singularity, then the Kähler-Ricci flow converges smoothly outside finitely many distinct holomorphic spheres with self-intersection number $(-1)$, and these $(-1)$ curves are contracted to distinct points in the Gromov-Hausdorff sense. Furthermore, making use of the work of [SoT3], it was shown that the flow can be extended on the blown-down surfaces, continuously in the Gromov-Hausdorff topology, and

Research supported in part by NSF grants DMS-1406124 and DMS-1406164. 
uniquely at the level of potentials. These geometric blow-down procedures terminate in finite time. Higher dimensional metric surgeries via the KählerRicci flow are constructed for certain families of projective manifolds in [SY, S] using a construction of birational cobordism in W].

Geometric convergence of the Kähler-Ricci flow at infinite time is still largely open. It is conjectured in SoT3 that if the Kähler-Ricci flow exists for all time, the normalized solution will either converge to a Kähler-Einstein metric space with possible singularities (see [EGZ]) or to a twisted KählerEinstein metric space of lower dimension, in the Gromov-Hausdorff topology. In particular, such spaces are expected to be homeomorphic to the canonical models of the original manifolds. This is known to hold if the manifold $X$ admits a Kähler-Einstein metric [C] or if there is a holomorphic submersion $\pi: X \rightarrow B$ with $c_{1}(B)<0$ and smooth Calabi-Yau fibers (established in [TWY], building on earlier work of [SoT1, SW3, GTZ, FZ, G]). For more general Kähler manifolds for which the flow exists for all time, weak analytic convergence is proved in [Ts, TZo when $X$ is of general type and in [SoT1, SoT2] when the flow collapses at infinity. In both cases, the scalar curvature of the normalized solutions of the Kähler-Ricci flow is always uniformly bounded [Z2, SoT4]. However, the problem of global geometric convergence is still open. One of the major obstacles is to obtain a uniform diameter bound for the normalized Kähler-Ricci flow.

In this paper, we will consider minimal surfaces of general type. By definition, a minimal surface of general type is a smooth complex surface $X$ whose canonical bundle $K_{X}$ is big and nef. Equivalently, there exists a smooth closed $(1,1)$-form $\chi$ in the first Chern class $c_{1}(X)$ such that $\chi \geq 0$ and $\int_{X} \chi^{2}>0$. In particular, $X$ is projective and so it is Kähler. Since there are no $(-1)$ curves on such surfaces, the Kähler-Ricci flow has long time existence and it is proved in [TZO that the normalized solution will converge weakly to the pullback of the unique orbifold Kähler-Einstein metric on the canonical model of $X$. More precisely, we will consider the pluricanonical system of $X$. For sufficiently large $m$, the holomorphic sections of $K_{X}^{m}$ induce a holomorphic map $\Phi: X \rightarrow \mathbb{P}^{N}$ with image $X_{\text {can }}$, the canonical model of $X$. $X_{\text {can }}$ is an algebraic surface with, at worst, finitely many orbifold $A$ $D$ - $E$-singularities (cf. [BHPV]). Moreover, $X_{\text {can }}$ admits a unique orbifold Kähler-Einstein metric Kob because $K_{X_{\text {can }}}$ is ample, which we will denote by $\omega_{\mathrm{KE}}$. The map $\Phi$ contracts $(-2)$ curves on $X$ to orbifold points on $X_{\text {can }}$.

We consider a solution $\omega(t)$ to the normalized Kähler-Ricci flow on $X$ starting at any Kähler metric $\omega_{0}$. Namely, $\omega=\omega(t)$ solves

$$
\frac{\partial}{\partial t} \omega=-\operatorname{Ric}(\omega)-\omega,\left.\quad \omega\right|_{t=0}=\omega_{0} .
$$

A unique solution to (1.1) exists for all time [C, Ts. It is known by the work of Tsuji $[\mathrm{TS}$ and Tian-Zhang [TZO that as $t \rightarrow \infty$, the solution $\omega(t)$ converges in $C^{\infty}$ on compact subsets of $X \backslash D$ to $\Phi^{*} \omega_{\mathrm{KE}}$, where $D$ is the union of the $(-2)$ curves on $X$. 
Our result concerns the global Gromov-Hausdorff behavior of $\omega(t)$ as $t \rightarrow \infty$. When $X$ only contains disjoint irreducible $(-2)$-curves, it was shown in SW4 that the evolving metrics of the normalized Kähler-Ricci flow converge to the unique Kähler-Einstein orbifold metric on $X_{\text {can }}$ in the Gromov-Hausdorff topology. However, when the $(-2)$ curve is not irreducible, the local metric models for the corresponding contracted $A-D-E$ singularities are usually complicated and not explicit, and so the arguments in SW2, SW4 cannot be immediately applied.

The following is our main result. We establish the global geometric convergence for the normalized Kähler-Ricci flow on all minimal surfaces of general type starting with any initial Kähler metric.

Theorem 1.1. Let $X$ be a minimal surface of general type and $\omega(t)$ be the solution of the normalized Kähler-Ricci flow (1.1). As $t \rightarrow \infty$, the Kähler manifolds $(X, \omega(t))$ converge in the Gromov-Hausdorff sense to $\left(X_{\mathrm{can}}, \omega_{\mathrm{KE}}\right)$. In particular, the diameter of $(X, \omega(t))$ is uniformly bounded for all $t$ and the convergence is smooth on the smooth part of $X_{\mathrm{can}}$.

The local smooth convergence was already proved in [Ts, TZo away from the $(-2)$ curves on $X$. Theorem 1.1 implies that all the $(-2)$ curves on $X$ are contracted to orbifold points by the Kähler-Ricci flow as $t \rightarrow \infty$. More precisely, if $E$ is a connected $(-2)$ curve on $X$, then for any $\varepsilon>0$, there exists an open neighborhood $U_{E, \varepsilon}$ of $E$ in $X$ such that the diameter of $U_{E, \varepsilon}$ with respect to the evolving metrics $\omega(t)$ is less than $\varepsilon$ for all sufficiently large $t$. Our approach does not rely on the a priori existence of the limiting orbifold Kähler-Einstein metric on $X_{\text {can }}$ constructed in Kob].

A surface of general type is a complex surface whose minimal model is a minimal surface of general type. Therefore, a surface of general type can be obtained by blowing up a minimal surface of general type finitely many times. Combined with the results of [SW2, SW4], we obtain:

Corollary 1.1. Let $X$ be a general type surface. Then the normalized Kähler-Ricci flow on $X$ starting with any initial Kähler metric $g_{0}$ is continuous through finitely many contraction surgeries in the Gromov-Hausdorff topology for $t \in[0, \infty)$ and converges in the Gromov-Hausdorff topology to $\left(X_{\mathrm{can}}, g_{\mathrm{KE}}\right)$. The convergence is smooth away from the $(-2)$-curves, where $g_{\mathrm{KE}}$ is the unique orbifold Kähler-Einstein metric on $X_{\mathrm{can}}$.

We refer the readers to [SW4, Theorem 1.1] for details of the canonical surgical contractions by the Kähler-Ricci flow.

As this paper was nearing completion, the authors became aware of a preprint of Gang Tian and Zhenlei Zhang [TZl] where the $L^{p}$ CheegerColding-Tian theory is used to obtain related results. We remark that the estimates in our proof do not require the a priori existence of the orbifold Kähler-Einstein metric on the canonical model, and we only use the maximum principle and other elementary arguments. 
The authors thank the referee for a helpful comment on a previous version of this paper.

\section{A PRIORI ESTIMATES}

Let $X$ be a minimal surface of general type. Since $K_{X}$ is big and nef, the pluricanonical linear system $\left|m K_{X}\right|$ is base point free for sufficiently large $m \in \mathbb{Z}^{+}$and it induces a birational holomorphic map $\Phi_{m}=\left|m K_{X}\right|$ : $X \rightarrow X_{\text {can }}$. The sequence $\Phi_{m}$ stabilizes for sufficiently large $m$ and $\Phi=\Phi_{m}$ contracts all the $(-2)$-curves on $X$. In particular, $K_{X}=\Phi^{*} K_{X_{\text {can }}}$.

We first reduce the Kähler-Ricci flow to a parabolic complex MongeAmpère equation, as in [Ts, TZO, SW2]. Define a closed $(1,1)$ form $\chi$ on $X$ by

$$
\chi=\frac{1}{m} \Phi^{*} \omega_{\mathrm{FS}} \in c_{1}\left(K_{X}\right),
$$

where $\Phi: X \rightarrow X_{\text {can }} \subset \mathbb{P}^{N}$ is the map induced by holomorphic sections of $K_{X}^{m}$, and $\omega_{\mathrm{FS}}$ is the Fubini-Study metric on $\mathbb{P}^{N}$. Choose $\Omega$ to be the smooth volume form satisfying

$$
\chi=\frac{\sqrt{-1}}{2 \pi} \partial \bar{\partial} \log \Omega, \quad \int_{X} \Omega=\int_{X} \omega_{0}^{2}
$$

Define a family of reference Kähler metrics $\hat{\omega}=\hat{\omega}(t)$ on $X$ by

$$
\hat{\omega}=\chi+e^{-t}\left(\omega_{0}-\chi\right) .
$$

Then the Kähler-Ricci flow (1.1) is equivalent to the parabolic complex Monge-Ampère equation for $\varphi=\varphi(t)$ given by

$$
\frac{\partial \varphi}{\partial t}=\log \frac{\left(\hat{\omega}+\frac{\sqrt{-1}}{2 \pi} \partial \bar{\partial} \varphi\right)^{2}}{\Omega}-\varphi,\left.\quad \varphi\right|_{t=0}=0 .
$$

Namely, if $\varphi$ solves (2.1) then $\omega(t):=\hat{\omega}+\frac{\sqrt{-1}}{2 \pi} \partial \bar{\partial} \varphi$ solves (1.1). Conversely if we are given a solution to (1.1) then we can obtain a solution $\varphi=\varphi(t)$ of (2.1).

Let $D=\sum_{i=1}^{M} D_{i}$ be the $(-2)$ curves contracted by $\Phi$, where $D_{i}$ is an irreducible component of $D$ and $D_{i}$ is a smooth rational curve with $D_{i}$. $D_{i}=-2$. All components of $D$ meet transversally with each other (see [BHPV], for example). Since the support of $D$ is the exceptional locus of the pluricanonical map $\Phi$ and $K_{X}$ is big and nef, the Kodaira lemma implies that there exist $a_{i} \in \mathbb{Q}^{+}$for $i=1, \ldots, M$ such that the $\mathbf{R}$-divisor

$$
K_{X}-\varepsilon D^{\prime}
$$

is ample for all $\varepsilon \in(0,1)$, where $D^{\prime}=\sum_{i=1}^{M} a_{i} D_{i}$.

Choose $\sigma_{i} \in \mathcal{O}\left(D_{i}\right)$, a holomorphic section of the line bundle $\left[D_{i}\right]$ associated to the divisor $D_{i}$, vanishing to order 1 along $D_{i}$. We fix now $\varepsilon_{0} \in(0,1)$. Then there exist Hermitian metrics $h_{i}$ on $\left[D_{i}\right]$ such that

$$
\chi-\varepsilon_{0} R_{h} \geq c_{\varepsilon_{0}} \omega_{0},
$$


for some positive constant $c_{\varepsilon_{0}}$, where we are using the notation

$$
R_{h}:=-\frac{\sqrt{-1}}{2 \pi} \partial \bar{\partial} \log h=\sum_{i=1}^{M}-a_{i} \frac{\sqrt{-1}}{2 \pi} \partial \bar{\partial} \log h_{i},
$$

for the curvature form of the Hermitian metric $h=\otimes_{i}\left(h_{i}\right)^{a_{i}}$ on the Q-line bundle associated to $\left[D^{\prime}\right]=\sum_{i=1}^{M} a_{i}\left[D_{i}\right]$.

In the next lemma we gather together some a priori estimates which are already known by the work of Tsuji, Tian-Zhang and Zhang [Ts, TZo, Z1, Z2].

Lemma 2.1. There exist uniform constants $C>0$ and $\lambda>0$ such that the following hold.

(i) $|\varphi|+|\dot{\varphi}|+|R| \leq C$ on $X \times[0, \infty)$.

(ii) On $X \times[0, \infty)$, we have

$$
\omega(t) \leq C|\sigma|_{h}^{-2 \lambda} \omega_{0}
$$

where $|\sigma|_{h}^{2}=\prod_{i}\left|\sigma_{i}\right|_{h_{i}}^{2}$.

(iii) For any compact $K \subset X \backslash D$, there exists for every $\ell=0,1,2, \ldots$ a constant $C_{K, \ell}$ such that

$$
\|\varphi\|_{C^{\ell}(K)} \leq C_{K, \ell}
$$

Proof. Part (i) is due to Tian-Zhang and Zhang [TZo, Z1, Z2, and makes use of the pluripotential theory estimates of Kołodziej [Kol] (for a recent survey, see $[\mathrm{PSoS}])$. Part (ii) was shown by Tsuji [Ts]. Part (iii) can be proved using a Calabi-type third order estimate and then higher order estimates (see [PSeS] and [ShW], for example).

Next, we discuss the local model for an irreducible $(-2)$-curve in $X$, following the discussion in [SW4. Fix an irreducible (-2)-curve $D_{j}$ in $X$. Then there exists a holomorphic map $\Psi: X \rightarrow \mathbb{P}^{K}$ contracting $D_{j}$ whose image $Y:=\Psi(X) \subset \mathbb{P}^{K}$ is subvariety with single orbifold point $y=\Psi\left(D_{j}\right) \in Y$. Using the notation above, $h_{j}$ is a Hermitian metric on the line bundle $\left[D_{j}\right]$ and $\sigma_{j}$ is a holomorphic section of this line bundle, vanishing to order 1 along $D_{j}$.

Write $B \subset \mathbb{C}^{2}$ for the unit ball centered at the origin. Write $\tilde{B}=B / \mathbb{Z}_{2}$, where $\mathbb{Z}_{2}$ acts on $B$ by

$$
\left(z^{1}, z^{2}\right) \mapsto\left(-z^{1},-z^{2}\right) .
$$

We can then identify a neighborhood of the orbifold point $y \in Y$ with $\tilde{B}$. Write $\omega_{\text {Eucl }}$ for the Euclidean metric

$$
\omega_{\text {Eucl }}=\sqrt{-1} d z^{1} \wedge d \bar{z}^{1}+\sqrt{-1} d z^{2} \wedge d \bar{z}^{2}
$$

on $B$, which descends to give a smooth orbifold metric on $\tilde{B}$. Define $r$ by

$$
r=\sqrt{\left|z^{1}\right|^{2}+\left|z^{2}\right|^{2}}
$$


on $B$ (and also on $\tilde{B})$. The map $\Psi$ is a biholomorphism from $\Psi^{-1}(\tilde{B} \backslash\{0\})$ to $\tilde{B} \backslash\{0\}$. The curve $D_{j}$ is given by $\left\{\sigma_{j}=0\right\}$ and we have (cf. [SW4, Section 2])

$$
\left|\sigma_{j}\right|_{h_{j}}^{2}=r^{4}
$$

where we are identifying $r$ with its pull-back via $\Psi$. In what follows we will, without comment, identify via $\Psi$ the sets $\Psi^{-1}(\tilde{B} \backslash\{0\})$ and $\tilde{B} \backslash\{0\}$, and similarly for the various functions and $(1,1)$ forms on these sets. Recalling that $\omega_{0}$ is a fixed Kähler metric on $X$, we note that on $\tilde{B} \backslash\{0\}$ we have

$$
C^{-1} r^{2} \omega_{\text {Eucl }} \leq \omega_{0} \leq \frac{C}{r^{2}} \omega_{\text {Eucl }}
$$

Moreover, if we define the vector field $V$ on $B \backslash\{0\}$ by

$$
V=z^{1} \frac{\partial}{\partial z^{1}}+z^{2} \frac{\partial}{\partial z^{2}}
$$

then we have

$$
\frac{r^{4}}{C} \leq|V|_{\omega_{0}}^{2} \leq C r^{4}
$$

for a uniform $C>0$.

A final remark about the local model is that if $p$ is any point in $D_{j}$ then we can find holomorphic coordinates $u, v$ in a neighborhood $W$ of $p$, and centered at $p$, such that $D_{j} \cap W=\{u=0\}$ and the map $\Psi$ from $W$ to $\tilde{B}$ is given by $\left(z^{1}, z^{2}\right): W \rightarrow \tilde{B}$ where

$$
u=\left(z^{1}\right)^{2}, \quad v=\frac{z^{2}}{z^{1}} .
$$

Namely, $z^{1}=u^{1 / 2}$ and $z^{2}=u^{1 / 2} v$, which gives a well-defined map into $\tilde{B}$.

Our next lemma shows that we have estimates for the evolving metric $\omega(t)$ on $\tilde{B} \backslash\{0\}$. However, unlike in [SW4], our $(-2)$-curve $D_{j}$ may intersect other $(-2)$ curves. To begin with, we only obtain estimates away from these intersection points. To make this more precise, fix any open tubular neighborhood $U_{\eta}^{j}$ of radius $\eta>0$, with respect to $\omega_{0}$, in $X$ of $\bigcup_{i \neq j} D_{i}$. This open set $U_{\eta}^{j}$ may intersect $\tilde{B} \backslash\{0\}$.

We have the following estimates, which are analogous to those of Lemma 4.1 and Lemma 10.1 in SW4.

Lemma 2.2. Fix $j \in\{1, \ldots, M\}$ and $\eta>0$. With the notation above, there exist constants $C$ and $\delta>0$ such that on $(\tilde{B} \backslash\{0\}) \cap\left(X-U_{\eta}^{j}\right)$, depending only on the fixed initial data and $\eta>0$ such that

(i) $\omega \leq \frac{C}{r^{2}} \omega_{\text {Eucl }}$.

(ii) $\omega \leq \frac{C}{r^{2(1-\delta)}}\left(\omega_{0}+\omega_{\text {Eucl }}\right)$.

(iii) $|V|_{\omega}^{2} \leq C r^{4 / 3}$, where $V$ is the vector field defined by (2.5). 
Proof. For simplicity of notation, we take $j=1$. The proof is fairly straightforward, given the arguments of [SW2, SW4]. The idea is to modify the quantities in SW4 by adding a term which tends to negative infinity along the union of $D_{2}, \ldots, D_{M}$. After applying the maximum principle, we will obtain a bound away from $U_{\eta}^{1}$.

Define on $\tilde{B} \backslash\{0\}$, for $\varepsilon>0$ and $A>>1$ a large constant to be determined,

$$
H_{\varepsilon}=\log \operatorname{tr}_{\omega_{0}} \omega+A \log \left(\left|\sigma_{1}\right|_{h_{1}}^{(1+\varepsilon)}\left(\prod_{i=2}^{M}\left|\sigma_{i}\right|_{h_{i}}^{4 \lambda}\right) \operatorname{tr}_{\omega_{\text {Eucl }}} \omega\right)-A^{2} \varphi,
$$

where $\lambda$ is the constant in item (ii) of Lemma 2.1. We wish to use the maximum principle to bound $H_{\varepsilon}$ from above on $\tilde{B} \backslash\{0\}$. Using Lemma 2.1. (ii) and the fact that the curve $D_{1}$ is completely contained inside $\tilde{B}$, we see that the quantity $H_{\varepsilon}$ is uniformly bounded from above on the boundary of $\tilde{B}$. In addition, we see from (2.4) that $H_{\varepsilon}$ tends to $-\infty$ as $r$ tends to 0 , and $H_{\varepsilon}$ tends to $-\infty$ on any curves $D_{2}, \ldots, D_{M}$ which intersect $\tilde{B}$. We also have an upper bound for $H_{\varepsilon}$ at time $t=0$. We may suppose then that $H_{\varepsilon}$ attains a maximum at an interior point $\left(x_{0}, t_{0}\right) \in(\tilde{B} \backslash\{0\}) \times(0, \infty)$, and $x_{0}$ does not lie on any of the curves $D_{2}, \ldots, D_{M}$. It suffices to bound $H_{\varepsilon}$ at this point $\left(x_{0}, t_{0}\right)$.

First recall the following well-known pointwise differential inequality for a solution $\omega=\omega(t)$ of the Kähler-Ricci flow [Y, A, C] (or see [SW3, Prop. 3.2.5] for a recent exposition)

$$
\left(\frac{\partial}{\partial t}-\Delta\right) \log \operatorname{tr}_{\beta} \omega \leq C_{\beta} \operatorname{tr}_{\omega} \beta-1,
$$

for a fixed metric $\beta$, where $-C_{\beta}$ is the infimum of the bisectional curvature of $\beta$. In particular, if the curvature of $\beta$ vanishes then we can take $C_{\beta}=0$.

At $\left(x_{0}, t_{0}\right)$, we have, applying (2.8) twice, first with $\beta=\omega_{0}$ and then with $\beta=\omega_{\text {Eucl }}$,

$$
\begin{aligned}
0 \leq & \left(\frac{\partial}{\partial t}-\Delta\right) H_{\varepsilon} \\
\leq & C_{0} \operatorname{tr}_{\omega} \omega_{0}+A\left(\frac{1+\varepsilon}{2}\right) \operatorname{tr}_{\omega} R_{h_{1}}+2 A \lambda \operatorname{tr}_{\omega}\left(\sum_{i=2}^{M} R_{h_{i}}\right) \\
& -A^{2} \dot{\varphi}+2 A^{2}-A^{2} \operatorname{tr}_{\omega} \hat{\omega} \\
= & C_{0} \operatorname{tr}_{\omega} \omega_{0}-A^{2} \dot{\varphi}+2 A^{2} \\
& -A^{2} \operatorname{tr}_{\omega}\left(\hat{\omega}-A^{-1}\left(\frac{1+\varepsilon}{2}\right) R_{h_{1}}-2 A^{-1} \lambda \sum_{i=2}^{M} R_{h_{i}}\right),
\end{aligned}
$$

where $-C_{0}$ is the lower bound for the bisectional curvature of $\omega_{0}$. Using (2.2) and the definition of $\hat{\omega}$, we may choose $A$ uniformly large enough such 
that, for a uniform $c_{0}>0$,

$$
\hat{\omega}-A^{-1}\left(\frac{1+\varepsilon}{2}\right) R_{h_{1}}-2 A^{-1} \lambda \sum_{i} R_{h_{i}} \geq c_{0} \omega_{0}
$$

and $A^{2} c_{0} \geq C_{0}+1$. Hence, using Lemma 2.1, part (i), we have

$$
0 \leq-\operatorname{tr}_{\omega} \omega_{0}-A^{2} \dot{\varphi}+C A^{2}
$$

for a uniform constant C. Now recall from Lemma 2.1 that $\varphi$ and $\dot{\varphi}$ (and hence also $\left.\log \left(\omega^{2} / \omega_{0}^{2}\right)\right)$ are uniformly bounded. It follows that we obtain a uniform upper bound of $\operatorname{tr}_{\omega} \omega_{0}$ at $\left(x_{0}, t_{0}\right)$, and from the inequality $\operatorname{tr}_{\omega_{0}} \omega \leq$ $\left(\operatorname{tr}_{\omega} \omega_{0}\right) \frac{\omega^{2}}{\omega_{0}^{2}}$, we also get an upper bound for $\operatorname{tr}_{\omega_{0}} \omega$. Then $H_{\varepsilon}$ is bounded from above at $\left(x_{0}, t_{0}\right)$, and hence has a uniform upper bound on $(\tilde{B} \backslash\{0\}) \times[0, \infty)$. Moreover, our upper bound is independent of $\varepsilon$.

Letting $\varepsilon \rightarrow 0$, we obtain on $\tilde{B} \backslash\{0\})$,

$$
\left(\operatorname{tr}_{\omega_{0}} \omega\right)^{1 / A}\left|\sigma_{1}\right|_{h_{1}}\left(\prod_{i=2}^{M}\left|\sigma_{i}\right|_{h_{i}}^{4 \lambda}\right) \operatorname{tr}_{\omega_{\mathrm{Eucl}}} \omega \leq C .
$$

First, using (2.4) we have $\left|\sigma_{1}\right|_{h_{1}} \operatorname{tr}_{\omega_{\text {Eucl }}} \omega \leq C \operatorname{tr}_{\omega_{0}} \omega$ and hence

$$
\operatorname{tr}_{\omega_{\text {Eucl }}} \omega \leq \frac{C}{r^{2}}\left(\prod_{i=2}^{M}\left|\sigma_{i}\right|_{h_{i}}^{4 \lambda}\right)^{-A /(A+1)}
$$

where we have used $\left|\sigma_{1}\right|_{h_{1}}=r^{2}$ from (2.3). We then obtain the estimate (i) since the quantity $\left(\prod_{i=2}^{M}\left|\sigma_{i}\right|_{h_{i}}^{2}\right)^{-1}$ is uniformly bounded on $X \backslash U_{\eta}^{1}$, depending only on the fixed data and $\eta$.

For (ii), observe that from (2.9) we also have

$$
\left(\operatorname{tr}_{\omega_{0}+\omega_{\text {Eucl }}} \omega\right)^{1+1 / A} \leq C\left|\sigma_{1}\right|_{h_{1}}^{-1}\left(\prod_{i=2}^{M}\left|\sigma_{i}\right|_{h_{i}}^{4 \lambda}\right)^{-1},
$$

and so using (2.3) again,

$$
\operatorname{tr}_{\omega_{0}+\omega_{\text {Eucl }}} \omega \leq C r^{-2(1-\delta)}\left(\prod_{i=2}^{M}\left|\sigma_{i}\right|_{h_{i}}^{4 \lambda}\right)^{-\frac{A}{A+1}}
$$

for some $\delta \in(0,1)$ depending only on $A$. This completes the proof of (ii).

For (iii), we will apply the maximum principle to $G_{\varepsilon}$, for $\varepsilon>0$, defined by

$$
G_{\varepsilon}=\log \left(|V|_{\omega}^{1+\varepsilon}\left(\prod_{i=2}^{M}\left|\sigma_{i}\right|_{h_{i}}^{4 \lambda}\right) \operatorname{tr}_{\omega_{\text {Eucl }}} \omega\right)+A\left(\left|z^{1}\right|^{2}+\left|z^{2}\right|^{2}\right),
$$

on $\tilde{B} \backslash\{0\}$, for $A>0$ to be determined. Note that from the bounds (2.3), (2.4) and (2.6), we see that for each fixed $t$, the quantity $G_{\varepsilon}(x)$ tends to $-\infty$ as $x$ approaches zero. Suppose that $G_{\varepsilon}$ achieves a maximum at a point 
$\left(x_{0}, t_{0}\right) \in(\tilde{B} \backslash\{0\}) \times(0, \infty)$ such that $x_{0}$ does not lie on any of the curves $D_{2}, \ldots, D_{M}$.

Following the computation of [SW2, Lemma 2.6], we compute at $\left(x_{0}, t_{0}\right)$,

$$
0 \leq\left(\frac{\partial}{\partial t}-\Delta\right) G_{\varepsilon} \leq \operatorname{tr}_{\omega}\left(\sum_{i=2}^{M} 2 \lambda R_{h_{i}}-A \omega_{\text {Eucl }}\right)<0
$$

if $A$ is chosen sufficiently large. This is a contradiction, implying that the maximum of $G_{\varepsilon}$ occurs at $t=0$ or on the boundary of $\tilde{B}$, where, by Lemma 2.1. we have the upper bound for $G_{\varepsilon}$. Thus $G_{\varepsilon}$ is bounded from above on $\tilde{B} \backslash\{0\}$, and moreover the bound is independent of $\varepsilon$. Letting $\varepsilon \rightarrow 0$, we obtain

$$
|V|{ }_{\omega} \operatorname{tr}_{\omega_{\text {Eucl }}} \omega \leq C\left(\prod_{i=2}^{M}\left|\sigma_{i}\right|_{h_{i}}^{4 \lambda}\right)^{-1} .
$$

But on $\left(X \backslash U_{\eta}^{1} \cap(\tilde{B} \backslash\{0\})\right.$, we have from (i),

$$
|V|_{\omega} \operatorname{tr}_{\omega_{\text {Eucl }}} \omega \leq C
$$

On the other hand,

$$
|V|_{\omega}^{2} \leq\left(\operatorname{tr}_{\omega_{\text {Eucl }}} \omega\right)|V|_{\omega_{\text {Eucl }}}^{2}=r^{2} \operatorname{tr}_{\omega_{\text {Eucl }}} \omega
$$

and multiplying both sides by $|V|_{\omega}$ gives $|V|_{\omega}^{2} \leq C r^{4 / 3}$ on $\left(X \backslash U_{\eta}^{1} \cap(\tilde{B} \backslash\{0\})\right.$. This completes the proof of the lemma.

As an immediate consequence of these estimates, we can obtain the following bound on the evolving metric $\omega=\omega(t)$ in terms of local holomorphic coordinate systems on $X$.

Corollary 2.1. Fix $j \in\{1, \ldots, M\}$ and $\eta>0$. We can cover $D_{j} \cap\left(X \backslash U_{\eta}^{j}\right)$ by finitely many complex coordinate charts $W$ with complex coordinates $u, v$ so that

(i) On each $W$, the curve $D_{j}$ is given by $D_{j}=\{u=0\}$.

(ii) There exists a uniform $C$ such that on each $W$,

$$
\omega(t) \leq C\left(\frac{\sqrt{-1} d u \wedge d \bar{u}}{|u|^{4 / 3}}+\sqrt{-1} d v \wedge d \bar{v}\right)
$$

Proof. By compactness, it suffices to prove the result for some neighborhood of a fixed point $p$ in $D_{j} \cap\left(X \backslash U_{\eta}^{j}\right)$. By the discussion above the statement of Lemma 2.2, we can find coordinates $u, v$ in a neighborhood $W$ of $p$ such 
that $W \cap D_{j}=\{u=0\}$ and $u, v$ satisfy (2.7). Compute on $W \backslash D_{j}$

$$
\begin{aligned}
\sqrt{-1}\left(d z^{1} \wedge d \bar{z}^{1}+d z^{2} \wedge d \bar{z}^{2}\right)= & \frac{\left(1+|v|^{2}\right) \sqrt{-1} d u \wedge d \bar{u}}{4|u|}+\sqrt{-1}|u| d v \wedge d \bar{v} \\
& +\operatorname{Re}\left(\sqrt{-1}\left(\frac{\bar{u}}{u}\right)^{1 / 2} v d u \wedge d \bar{v}\right) \\
\leq & C\left(\frac{\sqrt{-1} d u \wedge d \bar{u}}{|u|}+\sqrt{-1}|u| d v \wedge d \bar{v}\right),
\end{aligned}
$$

if we assume that $v$ is sufficiently small. Moreover, we have

$$
r^{2}=\left|z^{1}\right|^{2}+\left|z^{2}\right|^{2}=|u|\left(1+|v|^{2}\right) .
$$

Then the estimate (i) of Lemma 2.2 implies that on $W$ we have

$$
\omega \leq C\left(\frac{\sqrt{-1} d u \wedge d \bar{u}}{|u|^{2}}+\sqrt{-1} d v \wedge d \bar{v}\right)
$$

for a uniform constant $C$.

We can improve the bound in the $\partial / \partial u$ direction. Indeed, note that

$$
u \frac{\partial}{\partial u}=\frac{z^{1}}{2} \frac{\partial}{\partial z^{1}}+\frac{z^{2}}{2} \frac{\partial}{\partial z^{2}}=\frac{V}{2},
$$

and hence from part (iii) of Lemma 2.2 and (2.11),

$$
g\left(\frac{\partial}{\partial u}, \frac{\partial}{\partial \bar{u}}\right)=\frac{1}{4|u|^{2}}|V|_{\omega}^{2} \leq \frac{C r^{4 / 3}}{|u|^{2}} \leq \frac{C^{\prime}}{|u|^{4 / 3}},
$$

and this gives the estimate (ii) on $W$, as required.

Note that although the estimate (2.10) blows up as we approach $\{u=0\}$, it is strong enough to show that the diameter of each set $U_{\eta}^{j}$ is uniformly bounded. However at the moment, we have no estimates in neighborhoods of the intersection points $p_{i j}=D_{i} \cap D_{j}$ for $i \neq j$. The following lemma shows that our estimates do indeed extend to such neighborhoods.

Lemma 2.3. Fix an intersection point $p_{i j}=D_{i} \cap D_{j}$ for $i \neq j$. There exists a complex coordinate chart $W$ centered at $p_{i j}$ with complex coordinates $u, v$, such that $D_{i} \cap W=\{u=0\}$ and $D_{j} \cap W=\{v=0\}$. On $W \backslash\left(D_{i} \cup D_{j}\right)$, we have

$$
\omega(t) \leq C\left(\frac{\sqrt{-1} d u \wedge d \bar{u}}{|u|^{4 / 3}}+\frac{\sqrt{-1} d v \wedge d \bar{v}}{|v|^{4 / 3}}\right) .
$$

for a uniform constant $C$.

Proof. Since $D_{i} \cdot D_{j}=1$ if $i \neq j$ and $D_{i}$ and $D_{j}$ meet transversally, we can always find local complex coordinate chart $W$ centered at $p_{i j}$ with complex coordinates $u, v$, such that $D_{i} \cap W=\{u=0\}$ and $D_{j} \cap W=\{v=0\}$.

Write

$$
\omega_{\mathrm{con}}=\frac{\sqrt{-1} d u \wedge d \bar{u}}{|u|^{4 / 3}}+\frac{\sqrt{-1} d v \wedge d \bar{v}}{|v|^{4 / 3}} .
$$


Note that $\omega_{\text {con }}$ is a metric with conical singularities with simple normal crossings. The cone angle along $\left(D_{i} \cup D_{j}\right) \backslash\left\{p_{i j}\right\}$ is $2 \pi / 3$ (cf. [D, DGSW]). A key point is that on $W \backslash\left(D_{i} \cup D_{j}\right)$, the curvature of $\omega_{\text {con }}$ vanishes, as can be readily checked by a direct computation, or alternatively, one can apply change of variables by letting $u^{\prime}=u^{1 / 3}, v^{\prime}=v^{1 / 3}$ locally on a well-defined branch.

We will apply the maximum principle to the quantity $G=\log \operatorname{tr}_{\omega_{\text {con }}} \omega$ on $W \backslash\left(D_{i} \cup D_{j}\right)$. The estimates of Corollary 2.1 imply that the quantity $G$ is uniformly bounded from above on the boundary of the set $W$. Applying (2.8) with $\beta=\omega_{\text {con }}$ and $C_{0}=0$ we see that on $W \backslash\left(D_{i} \cup D_{j}\right)$ we have

$$
\left(\frac{\partial}{\partial t}-\Delta\right) G \leq-1
$$

which implies that the maximum of $G$ occurs either at $t=0$ or on the boundary of $W$. In both cases, we have a uniform upper bound 1 for $G$. Hence $G \leq C$ on $W \backslash\left(D_{i} \cup D_{j}\right)$, completing the proof of the lemma.

From the estimates now obtained, we can bound distances with respect to $g(t)$ in a neighborhood of the curve $D$. Denote by $B_{g_{0}, \delta}(p)$ the $g_{0}$ geodesic ball in $X$ centered at $p \in X$ with radius $\delta$, and by $\tilde{U}_{\delta}$ the $\delta$-tubular neighborhood of $D$ with respect to $g_{0}$,

$$
\tilde{U}_{\delta}:=\left\{x \in X \mid d_{g_{0}}(x, D)<\delta\right\} .
$$

Then we have:

Proposition 2.1. There exist uniform positive constants $C, \delta_{0}$ so that the following hold.

(i) For every intersection point $p_{i j}=D_{i} \cap D_{j}$, and any $\delta \in\left(0, \delta_{0}\right)$, we have

$$
\operatorname{diam}_{g(t)} B_{g_{0}, \delta}\left(p_{i j}\right) \leq C \delta^{1 / 3}
$$

(ii) For every $\delta \in\left(0, \delta_{0}\right)$,

$$
d_{g(t)}(D, q) \leq C \delta^{1 / 3}, \quad \text { for all } q \in \tilde{U}_{\delta} .
$$

Proof. For (i), fix an intersection point $p_{i j}$, and let $W$ be as in the statement of Lemma 2.3, with $p_{i j}$ corresponding to the point $u=v=0$ in the complex coordinates $u, v$. We assume that $\delta_{0}>0$ is sufficiently small so that $W$ contains the ball $B_{g_{0}, \delta_{0}}\left(p_{i j}\right)$. Given a point $\left(u_{0}, v_{0}\right) \in W$ with both $u_{0}$ and $v_{0}$ nonzero, we compute using the estimate (2.12) that the length of the path $\gamma:[0,1] \rightarrow W$ given by $\gamma(\lambda)=\left(\lambda u_{0}, \lambda v_{0}\right)$, with respect to $g(t)$, satisfies

$$
\text { Length }_{g(t)}(\gamma) \leq C\left(\left|u_{0}\right|^{1 / 3}+\left|v_{0}\right|^{1 / 3}\right) \leq C^{\prime} \delta^{1 / 3},
$$

for uniform constants $C, C^{\prime}$. Since for each $t$, the metric $\omega(t)$ is smooth, we obtain this bound even when one of $u_{0}, v_{0}$ is zero. This gives (i).

\footnotetext{
${ }^{1}$ To clarify this part of the argument (we thank Valentino Tosatti for pointing out our imprecision here): fix $\varepsilon>0$ and consider $G_{\varepsilon}=\log \operatorname{tr}_{\omega_{\text {con }}} \omega+\varepsilon \log \frac{\omega^{2}}{\omega_{\text {con }}^{2}}$ which satisfies $\left(\frac{\partial}{\partial t}-\Delta\right) G_{\varepsilon} \leq-1$. Then let $\varepsilon \rightarrow 0$.
} 
For (ii), let $q \in \tilde{U}_{\delta}$. First assume that $q$ is contained in a coordinate chart $W$ as in the discussion above, and $q$ has coordinates $\left(u_{0}, v_{0}\right)$. Since $q$ is in $\tilde{U}_{\delta}$, at least one of $\left|u_{0}\right|$ or $\left|v_{0}\right|$ is of order $\mathrm{O}(\delta)$. Without loss of generality we suppose that $\left|u_{0}\right|=\mathrm{O}(\delta)$ and $v_{0} \neq 0$. Then consider the path $\gamma:[0,1] \rightarrow W$ given by $\gamma(\lambda)=\left(\lambda u_{0}, v_{0}\right)$. The length of this path with respect to $g(t)$ satisfies

$$
\text { Length }_{g(t)}(\gamma) \leq C\left|u_{0}\right|^{1 / 3} \leq C^{\prime} \delta^{1 / 3}
$$

since when restricted to the $\partial / \partial u$ and $\partial / \partial \bar{u}$ directions, the metric $\omega(t)$ is bounded from above by $C \sqrt{-1} d u \wedge d \bar{u} /|u|^{4 / 3}$.

If $q \in \tilde{U}_{\delta}$ is not contained in such a neighborhood $W$ of an intersection point, we instead apply the estimate of Corollary 2.1, and we obtain the required bound by an identical argument. This completes the proof of (ii).

\section{Gromov-HausdorfF CONVERGence}

Given the estimates above, it is now straightforward to derive the GromovHausdorff convergence of $(X, g(t))$ to $\left(X_{\mathrm{can}}, g_{\mathrm{KE}}\right)$. First, we have

$$
\int_{D} \omega(t)=e^{-t} \int_{D} \omega_{0} \longrightarrow 0, \quad \text { as } t \rightarrow \infty
$$

where $D=\sum_{i} D_{i}$ is the $(-2)$ curve as introduced above. Indeed, this follows immediately from the Kähler-Ricci flow equation since

$$
\frac{\partial}{\partial t} \int_{D} \omega(t)=-\int_{D} \operatorname{Ric}(\omega(t))-\int_{D} \omega(t)=-\int_{D} \omega(t),
$$

where we have used the fact that $K_{X} \cdot D=0$.

First we prove:

Lemma 3.1. If $\hat{D}$ is a connected component of $D$ then

$$
\operatorname{diam}_{g(t)} \hat{D} \rightarrow 0, \quad \text { as } t \rightarrow \infty .
$$

Proof. For simplicity, assume that $\hat{D}=D_{1} \cup D_{2}$, and that $D_{1}$ and $D_{2}$ intersect at a point $p$ (the general case is similar). Fix $\varepsilon>0$. Then by Proposition 2.1 there exists $\delta>0$ such that the $g_{0}$ geodesic ball $B_{g_{0}, \delta}(p)$ of radius $\delta$ centered at $p$ has

$$
\operatorname{diam}_{g(t)} B_{g_{0}, \delta}(p)<\varepsilon / 3 .
$$

Define $D_{1}^{(\varepsilon)}=D_{1} \backslash B_{g_{0}, \delta}(p)$. The estimate of Lemma 2.2, part (ii), together with [SW4, Lemma 5.1] gives the existence of a constant $C_{\varepsilon}$, depending on $\varepsilon$, such that

$$
\left.\omega(t)\right|_{D_{1}^{(\varepsilon)}} \leq\left. C_{\varepsilon} \omega_{0}\right|_{D_{1}^{(\varepsilon)}} .
$$

We claim that if $a, b$ are any two points in $D_{1}^{(\varepsilon)}$, then

$$
d_{g(t)}(a, b) \leq C_{\varepsilon}^{\prime} e^{-t / 3},
$$


for another constant $C_{\varepsilon}^{\prime}$ depending on $\varepsilon$. The claim follows almost verbatim from the argument in [SW2] or [SSW, Lemma 2.4] and the estimate (3.1). Indeed, the only differences are that we have to keep track of the dependence on $\varepsilon$, and we replace the factor $(T-t)$ in [SSW, Lemma 2.4] with $e^{-t}$.

The proof is now essentially finished. Choose $t$ sufficiently large so that $C_{\varepsilon}^{\prime} e^{-t / 3}<\varepsilon / 3$, so that

$$
\operatorname{diam}_{g(t)} D_{1}^{(\varepsilon)} \leq \varepsilon / 3 .
$$

Similarly, if we set $D_{2}^{(\varepsilon)}=D_{2} \backslash B_{g_{0}, \delta}(p)$ then we obtain

$$
\operatorname{diam}_{g(t)} D_{2}^{(\varepsilon)} \leq \varepsilon / 3 \text {. }
$$

The result then follows by combining (3.2), (3.3) and (3.4).

Combining this lemma with the estimates established in Proposition 2.1, we immediately obtain:

Lemma 3.2. Let $\hat{D}$ be a connected component of $D$. For $\delta>0$, write

$$
\hat{U}_{\delta}:=\left\{x \in X \mid d_{g_{0}}(x, \hat{D})<\delta\right\},
$$

for its $\delta$-tubular neighborhood with respect to $g_{0}$. Then for any $\varepsilon>0$ there exists $\delta>0$ and $T>0$ such that

$$
\operatorname{diam}_{g(t)} \hat{U}_{\delta} \leq \varepsilon, \quad \text { for } t \geq T \text {. }
$$

As a consequence, the diameter of $(X, g(t))$ is uniformly bounded.

Proof. Combine part (ii) of Proposition 2.1 with Lemma 3.1 to obtain (3.5). For the last assertion, fix $\delta>0$ and use Lemma 2.1 to see that the diameter of $X \backslash V_{\delta}$ is uniformly bounded, where $V_{\delta}$ is the union of the $\delta$-tubular neighborhoods $\hat{U}_{\delta}$ of all the connected components of $D$.

Finally, we complete the proof of our main result.

Proof of Theorem 1.1. By the results in [Ts] and [TZO], the solution $\varphi(t)$ of the Monge-Ampère flow (2.1) converges smoothly on $X \backslash D$ to a bounded $\chi$-psh function $\varphi_{\mathrm{KE}}$ satisfying

$$
\left(\chi+\frac{\sqrt{-1}}{2 \pi} \partial \bar{\partial} \varphi_{\mathrm{KE}}\right)^{2}=e^{\varphi_{\mathrm{KE}} \Omega}
$$

as $t \rightarrow \infty$ and $\varphi_{\mathrm{KE}} \in C^{\infty}(X \backslash D)$. Since $\chi$ vanishes on $D$ and $\varphi_{\mathrm{KE}} \in$ $L^{\infty}(X) \cap \operatorname{PSH}(X, \chi), \varphi_{\mathrm{KE}}$ is constant along each connected component of $D$. Immediately $\chi+\frac{\sqrt{-1}}{2 \pi} \partial \bar{\partial} \varphi_{\mathrm{KE}}=\Phi^{*} \omega_{\mathrm{KE}}$ for some Kähler current $\omega_{\mathrm{KE}} \in$ $-c_{1}\left(X_{\text {can }}\right)$ on $X_{\text {can }}$ with bounded local potentials. We then can apply the smoothing properties of the weak Kähler-Ricci flow [SoT2, Theorem 4.5] on Kähler orbifolds to show that $\omega_{\mathrm{KE}}^{\prime}$ must be a smooth orbifold KählerEinstein metric on $X_{\text {can }}$. In particular, $\omega_{\mathrm{KE}}=\omega_{\mathrm{KE}}^{\prime}$ by the uniqueness of orbifold Kähler-Einstein metrics on Kähler orbifolds with ample canonical bundle. 
From the local smooth convergence of $\varphi$ to $\varphi_{\mathrm{KE}}$ on $X \backslash D$, we have

$$
\omega(t) \rightarrow \omega_{\mathrm{KE}}, \quad \text { as } t \rightarrow \infty,
$$

in $C^{\infty}(K)$ for any compact subsets $K \subset X \backslash D$. Combining this fact with Lemma 3.2 and the argument of [SW2, Section 3], we immediately obtain the Gromov-Hausdorff convergence of $(X, \omega(t))$ to $\left(X_{\mathrm{can}}, \omega_{\mathrm{KE}}\right)$.

\section{REFERENCES}

[A] Aubin, T. Equations du type Monge-Ampère sur les variétés kählériennes compactes, Bull. Sci. Math. (2) 102 (1978), no. 1, 63-95

[BHPV] Barth, W. P., Hulek, K., Peters, C. A. M. and Van de Ven, A. Compact complex surfaces. Second edition. Ergebnisse der Mathematik und ihrer Grenzgebiete. Springer-Verlag, Berlin

[C] Cao, H.-D. Deformation of Kähler metrics to Kähler-Einstein metrics on compact Kähler manifolds, Invent. Math. 81 (1985), no. 2, 359-372

[CT] Collins, T. and Tosatti, V. Kähler currents and null loci, preprint, arXiv:1304.5216

[DGSW] Datar, V., Guo, B., Song, J. and Wang, X. Connecting toric conical KählerEinstein manifolds, arXiv:1308.6781

[D] Donaldson, S.K. Kähler metrics with cone singularities along a divisor, Essays in mathematics and its applications, 49-79, Springer, Heidelberg, 2012.

[EGZ] Eyssidieux, P., Guedj, V. and Zeriahi, A. Singular Kähler-Einstein metrics. J. Amer. Math. Soc. 22 (2009), no. 3, 607-639

[FIK] Feldman, M., Ilmanen, T. and Knopf, D. Rotationally symmetric shrinking and expanding gradient Kähler-Ricci solitons, J. Differential Geometry 65 (2003), no. 2, 169-209

[FZ] Fong, F. T.-H. and Zhang, Z. The collapsing rate of the Kähler-Ricci flow with regular infinite time singularity, J. Reine Angew. Math. 703 (2015), 95-113

[G] Gill, M. Collapsing of products along the Kähler-Ricci flow, Trans. Amer. Math. Soc. 366 (2014), no. 7, 3907-3924

[GTZ] Gross, M., Tosatti, V., Zhang, Y. Collapsing of abelian fibred Calabi-Yau manifolds, Duke Math. J. 162 (2013), no. 3, 517-551

[H] Hamilton, R. S. Three-manifolds with positive Ricci curvature, J. Differential Geom. 17 (1982), no. 2, 255-306

[Kob] Kobayashi, R., Einstein-Kähler V-metrics on open Satake V-surfaces with isolated quotient singularities, Math. Ann. 272 (1985), no. 3, 385-398

[Kol] Kołodziej, S. The complex Monge-Ampère equation, Acta Math. 180 (1998), no. 1, 69-117

[P1] Perelman, G. The entropy formula for the Ricci flow and its geometric applications, preprint, arXiv: math.DG/0211159

[P2] Perelman, G. Ricci Flow with Surgery on Three-Manifolds, preprint, arXiv: math.DG/0303109

[P3] Perelman, G. Finite Extinction Time for the Solutions to the Ricci Flow on Certain Three-Manifolds, preprint, arXiv: math.DG/0307245

[PSeS] Phong, D. H., Sesum, N. and Sturm, J. Multiplier ideal sheaves and the Khler-Ricci flow, Comm. Anal. Geom. 15 (2007), no. 3, 613-632

[PSoS] Phong, D.H., Song, J. and Sturm, J. Complex Monge-Ampère equations, Surveys in differential geometry Vol. XVII, 327-410, Surv. Differ. Geom., 17, Int. Press, Boston, MA, 2012.

[ShW] Sherman, M. and Weinkove, B. Interior derivative estimates for the Kähler-Ricci flow, Pacific J. Math. 257 (2012), no. 2, 491-501

[S] Song, J. Ricci flow and birational surgery, arXiv:1304.2607 
[SSW] Song, J., Székelyhidi, G. and Weinkove, B. The Kähler-Ricci flow on projective bundles, Int. Math. Res. Not. 2013 (2013), no. 2, 243-257

[SoT1] Song, J., and Tian, G. The Kähler-Ricci flow on surfaces of positive Kodaira dimension, Invent. Math. 170 (2007), no. 3, 609-653

[SoT2] Song, J., and Tian, G. Canonical measures and Kähler-Ricci flow, J. Amer. Math. Soc. 25 (2012), no. 2, 303-353

[SoT3] Song, J., and Tian, G. The Kähler-Ricci flow through singularities, preprint, arXiv: 0909.4898

[SoT4] Song, J., and Tian, G. Bounding scalar curvature for global solutions of the KählerRicci flow, arXiv:1111.5681, to appear in Amer. J. Math.

[SW1] Song, J. and Weinkove, B. The Kähler-Ricci flow on Hirzebruch surfaces, J. Reine Angew. Math. 659 (2011), 141-168

[SW2] Song, J. and Weinkove, B. Contracting exceptional divisors by the Kähler-Ricci flow, Duke Math. J. 162 (2013), no. 2, 367-415

[SW3] Song, J. and Weinkove, B. Introduction to the Kähler-Ricci flow, Chapter 3 of "Introduction to the Kähler-Ricci flow", eds S. Boucksom, P. Eyssidieux, V. Guedj, Lecture Notes Math. 2086, Springer 2013

[SW4] Song, J. and Weinkove, B. Contracting exceptional divisors by the Kähler-Ricci flow, Proc. Lond. Math. Soc. (3) 108 (2014), no. 6, 1529-1561

[SY] Song, J. and Yuan, Y. Metric flips with Calabi ansatz, Geom. Func. Anal. 22 (2012), no. 1, 240-265

[T] Tian, G. New results and problems on Kähler-Ricci flow, Géométrie différentielle, physique mathématique, mathématiques et société. II. Astérisque 322 (2008), 71-92

[TZl] Tian, G. and Zhang, Z. Convergence of the Kähler-Ricci flow on lower dimensional smooth minimal models of general type, preprint

[TZo] Tian, G. and Zhang, Z. On the Kähler-Ricci flow on projective manifolds of general type, Chinese Ann. Math. Ser. B 27 (2006), no. 2, 179-192

[TWY] Tosatti, V., Weinkove, B. and Yang, X. The Kähler-Ricci flow, Ricci-flat metrics and collapsing limits, preprint, arXiv: 1408.0161

[Ts] Tsuji, H. Existence and degeneration of Kähler-Einstein metrics on minimal algebraic varieties of general type, Math. Ann. 281 (1988), no. 1, 123-133

[W] Wlodarczyk, J. Birational cobordisms and factorization of birational maps, J. Algebraic Geom. 9 (2000), no. 3, 425-449

[Y] Yau, S.-T. On the Ricci curvature of a compact Käahler manifold and the complex Monge-Ampère equation, I, Comm. Pure Appl. Math. 31 (1978), no. 3, 339-411

[Z1] Zhang, Z. On degenerate Monge-Ampère equations over closed Kähler manifolds, Int. Math. Res. Not. 2006, Art. ID 63640, (2006) 18 pp

[Z2] Zhang, Z. Scalar curvature bound for Kähler-Ricci flows over minimal manifolds of general type, Int. Math. Res. Not. IMRN 2009 (2009), no. 20, 3901-3912

Department of Mathematics, Rutgers University, Piscataway, NJ 08854

Department of Mathematics, Rutgers University, Piscataway, NJ 08854

Department of Mathematics, Northwestern University, Evanston, IL 60208 